\title{
Apodization of Immersed Light Source for Layer Structures and Application for the Detailed Human Eye Modelling
}

\author{
Chung - Jen Ou ${ }^{1}$, Shan-En Hong ${ }^{2}$, Rebecca Han-Ying Sun ${ }^{3, *}$ \\ ${ }^{1}$ Hsiuping Solar Observatory, Electrical Engineering, Hsiuping University of Science and Technology, Taiwan \\ ${ }^{2}$ Department of Electrical Engineering, Hsiuping University of Science and Technology, Taiwan \\ ${ }^{3}$ School of Optometry, Chung Shan Medical University, Taiwan
}

Copyright $(\mathcal{C} 2015$ by authors, all rights reserved. Authors agree that this article remains permanently open access under the terms of the Creative Commons Attribution License 4.0 International License.

\begin{abstract}
Simulation of the immersed light source with microstructure on the emitting surface is critical for next generation optical and bio-photonics components. Several design procedures that corresponding to the modulation of the emitting pattern is addressed. The writers are at present operating on a MOST project "Investigation of Solid Human Eye Model with 3D Printing Technology and the Evaluation on the Associate Specialized Optoelectronic Sensing Structures Fabrication to the Biocompatible Surface". We are preparing the methodology that can construct the human eye model with immersed emitting energy by the $3 \mathrm{D}$ printing material, and furnishes the real time estimation on the image qualities of the human eye. Our aim is to build a practical platform to evaluate the immersed optical-electronic components of the human eye. By doing this, the evaluation of the visual qualities can be amended, and it becomes possible for us to fill in an accurate measurement system that based on the eye model with immersed source. In this account, we provide several comments on the conduct of the immersed light source and the application to the details human eye modeling.
\end{abstract}

Keywords Immersed, Human Eye, 3D Printing

\section{Introduction}

Layer structure with light source immersed in the material provides as important applications for information display [1-8]. One of the applications for such device is to develop the optoelectronic component for the building and evaluation on the metrics of the (artificial) human eye. It is anticipated that a flexible and emitting light source layer can be attached or embedded in the human eye for specific uses. Granting to the comments by ophthalmologists, we realized that the current clinicians often meet the difficulties to describe the visual improvement of the patients, before and after the discussion or the operating room. No question those expensive facilities can simultaneously assess the visual metrics of the patients. Yet, for the patients and relevant, it is not easy to understand these complicated medical conditions and phenomena. On the other hand, the specialist sometimes will be difficult to predict and interpreted the possible effects of the treatment, for the unexpected scenario that the patients will endure-through for their daily life (such as Halo, glare phenomena while driving under different visual/head position). Moreover, for the upcoming aging society, to depict the subjective visual experience of the patient may not be easy - wearable and immersed type device is possible one of the answers. Based on these demands, this report will pay attention on the ray tracing of the immersed layer device.

Referable to the complicated fabrication procedures for these novel devices, there are sometimes contradictions between the numerical simulation and the experiment report. For example, it is anticipated that the projected light source that immersed under the tissue, without textures on the top emitting surface should take in a flatten apodization for the smaller solid angle region, while the one with microstructure will lead to the Lambertain pattern (due to the increasing of the extraction efficiency). However, experimental results differ for single layer and multi-layers situations. This is imputable to the combinations between the refractive index, interface conditions and the surface properties for each layer that lead to these disagreements. Such divergence in the planning stage will cause serious and severe consequences for developing medical applications. To clarify such contradictions, we demonstrate the emitting patterns of a single layer immersed light source to prevent possible issues for practical application with 3D printing.

\section{Material}

Through the using of the 3D printing technologies, an accurate solid eye model with immersed light source for a 
specific patient can be fabricated by the specialist. We cooperate with the $3 \mathrm{D}$ printer fabricator, to evaluate and investigating the appropriate printing bio-compatible material. The evaluation also includes the performance of the optical element and the sensing circuits. To complete the reliable correlation between the material properties, it is critical to understand the metric of the image while these optical components are layered structure of the tissues of the human eye. Simulation on the Glaucoma, Cataracts and Age-Related Macular Degeneration (AMD) can be also complete through proper pigment injection and the pressurized of the example. During the designing of the complicated layer structure and the combinations of the light source for layer device, appropriate modelling is vital for the comparability between the experimental results and the designed human eye model. If the construction is planned under the immersed light source for information display or illuminating purpose, and so it is essential to interpret the light propagating phenomena between these optical systems. To investigate the effect, figure 1 is the basic model that a light source immersed in the material. $\mathrm{n}_{1}$ and $\mathrm{n}_{2}$ are the two refractive indices. A defined relative ratio $\Gamma \equiv \mathrm{n}_{2} / \mathrm{n}_{1}$ is introduced to observe the emitting radiant pattern (apodization) of the device. Note that this kind of configuration can be the adaptive light source that immersed in the specific bio-compatible material - while the refractive index can be modulated through the physical, electro-chemical effects, even mechanical treatment. Observe the light source can be charged up from the extraneous.

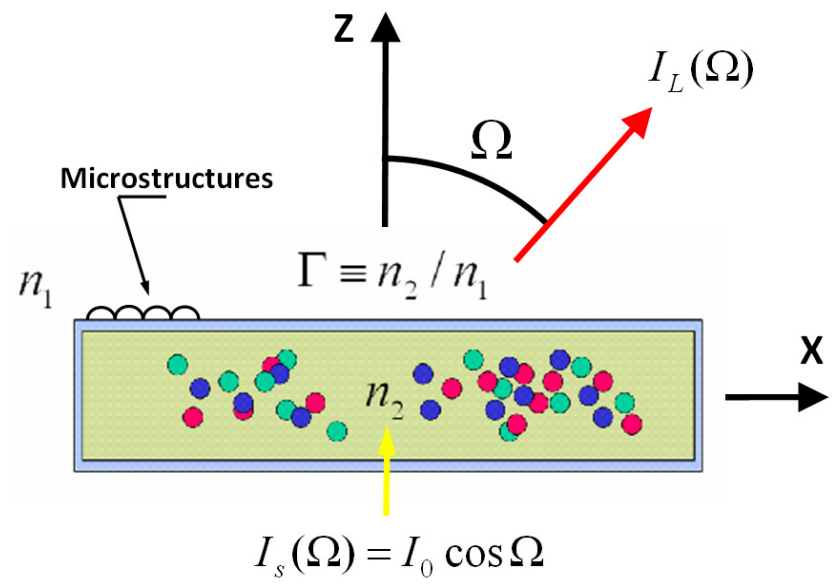

Figure 1. Concept for immersed light source model with particles and microstructures

The Intensity distribution of the light source (yellow color) is defined as the Lambertian model in the following configuration:

$$
I_{S}(\Omega)=I_{0} \cos \Omega
$$

where $I_{0}$ is the peak intensity of the apodization patterns, $\Omega$ is the spatial angle that measure from the $\mathrm{z}$ axis. After emitting out through the layer structure, the Intensity distribution $I_{\mathrm{L}}(\theta)$ will be transformed. Since the giving of power is set as 1 unit $(1 \mathrm{~W})$, therefore the followings should stand if one considers the possible power consumption inside the tissues.

$$
\int I_{s}(\Omega) d \Omega \geq \int I_{L}(\Omega) d \Omega
$$

\section{Results and Discussions}

During the simulation, an alternative that turns-on/off the immersed status is present by different simulators. By comparing the apodization of previous fundamental configuration shown in figure 1, we found that such option for the absorption of the light source of the fabric might not contribute to significant effects. However, this turn on procedure is important for the Boolean operation in the latter layer stacking sequences, since it is extremely important to indicate the properties of the material that encapsulates the light source. In the case of the human eye, the material can be the vitreous body or the lens. Meanwhile, as the ratio $\Gamma$ approach to 1 , the emitting patterns for this device return to the Lambertian type (figure 2). Notice that there are two intensity peaks occurring along 45 degrees, and then disappears during the increasing of the ratio $\Gamma$. The green circles in figure indicating the same intensity, as the scale measure for the comparison of the intensity distributions. As expected that if there are designed micro-structures or specific patterns on the top surface of the immersed light source, the extracting energy will occur. It is worth to mention that the fabrication of the bio-compatible microstructure on the tissue is one of the tasks in our project.

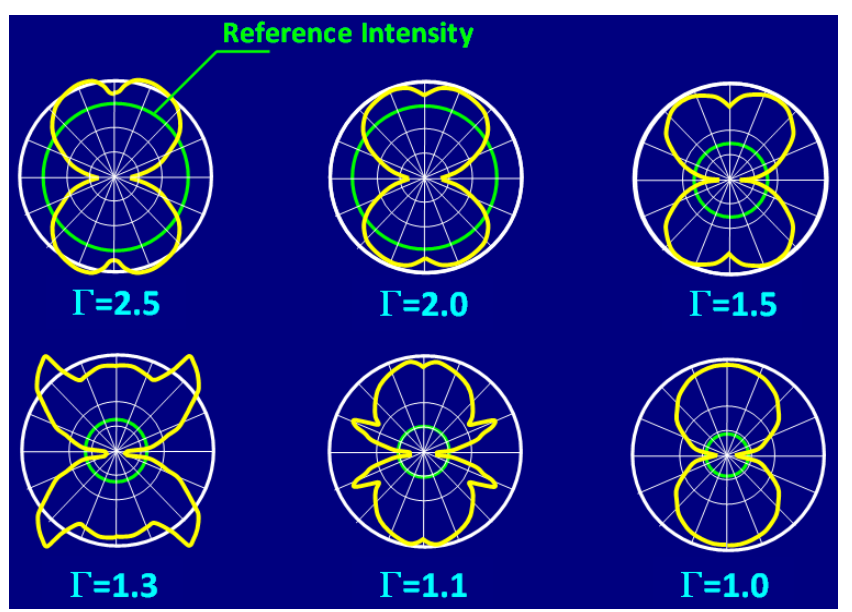

Figure 2. Scenario for Immersed type light source

Thus, through the careful combination between the two procedures (adjusting the $\Gamma$ ratio and the surface texture), the emitting radiant than can be accommodated to spread over several critical apodization - such as the uniform distribution along the target plane, the side emitting and the Lambertian type. Stand on these phenomena, the writers have already filed the patents with such simple concept for the information display and bionic and bio-compatible device. 
Still, the troubles with the delicate controlling of the refractive ratio and the cost efficient method to regulate it are indeed a challenging job.

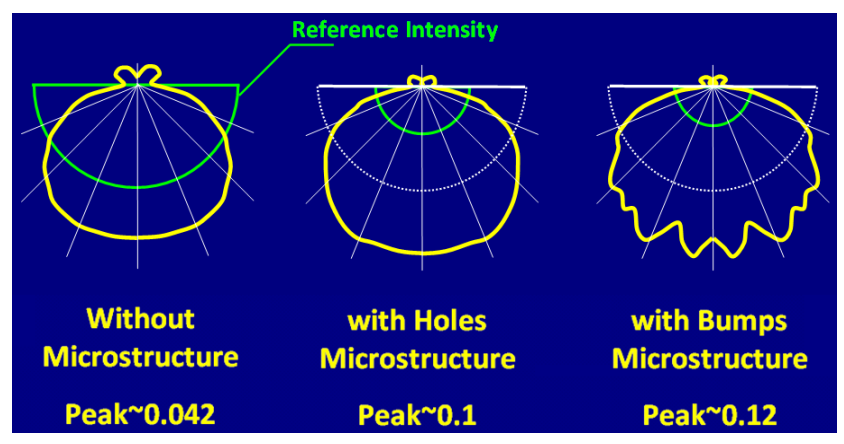

Figure 3. Effect of reflective layer to microstructures on the top surface of the source

Immediately one of the interesting things is the effects of the microstructure that build on the refracting layer of the gimmick. The microstructures can be helpful for the extraction of the power from the light source, and there are two strategies to manufacture it - bumps and hollows. Observe that the biological tissues also exhibit these microstructures that should sustain the significant importance during the evolution. 3D printing methods to fabricate the microstructures can be contained by the resolution and the filling pattern options. It is clear to see in figure 3 that almost all three types of configurations reveals the Lambertian-like pattern. With and without the surface structure indicates a 2.5 times extraction efficiency than the one without surface treatments. Moreover, the size of the micro-constructions (in this example a semi-spherical is introduced) is almost not relevant to the emitting patterns. This argues that the fabrication criterion for this type of gimmick is much gentler for a compact, portable/wearable and small size requirement. However, bumps and holes lead to slightly different in the small angle region. If one is to design the illuminating condition with more uniformity, the light source should be immersed under the hole type surface structures since there are less irregular peak patterns that shown in figure 3.

This formula can be applied to the provision of the wearable eye device (an eye model is depicted later in figure 4), since the emitting angle and the uniformity will related to the angle of the incident ray, which can be interpreted as the F-number, object Numerical Aperture (N.A.) and the pupil diameter of the human eye. On the other hand, the structure of the retina also demonstrated as layer structures [9], photons can be treated as emitting from specific layers for successive ray tracing to explore the efficiency and sensitivity of the retina. Such that the emitting patterns reveals in figure 2 and 3 provide the estimation on the power spreading of the photons.

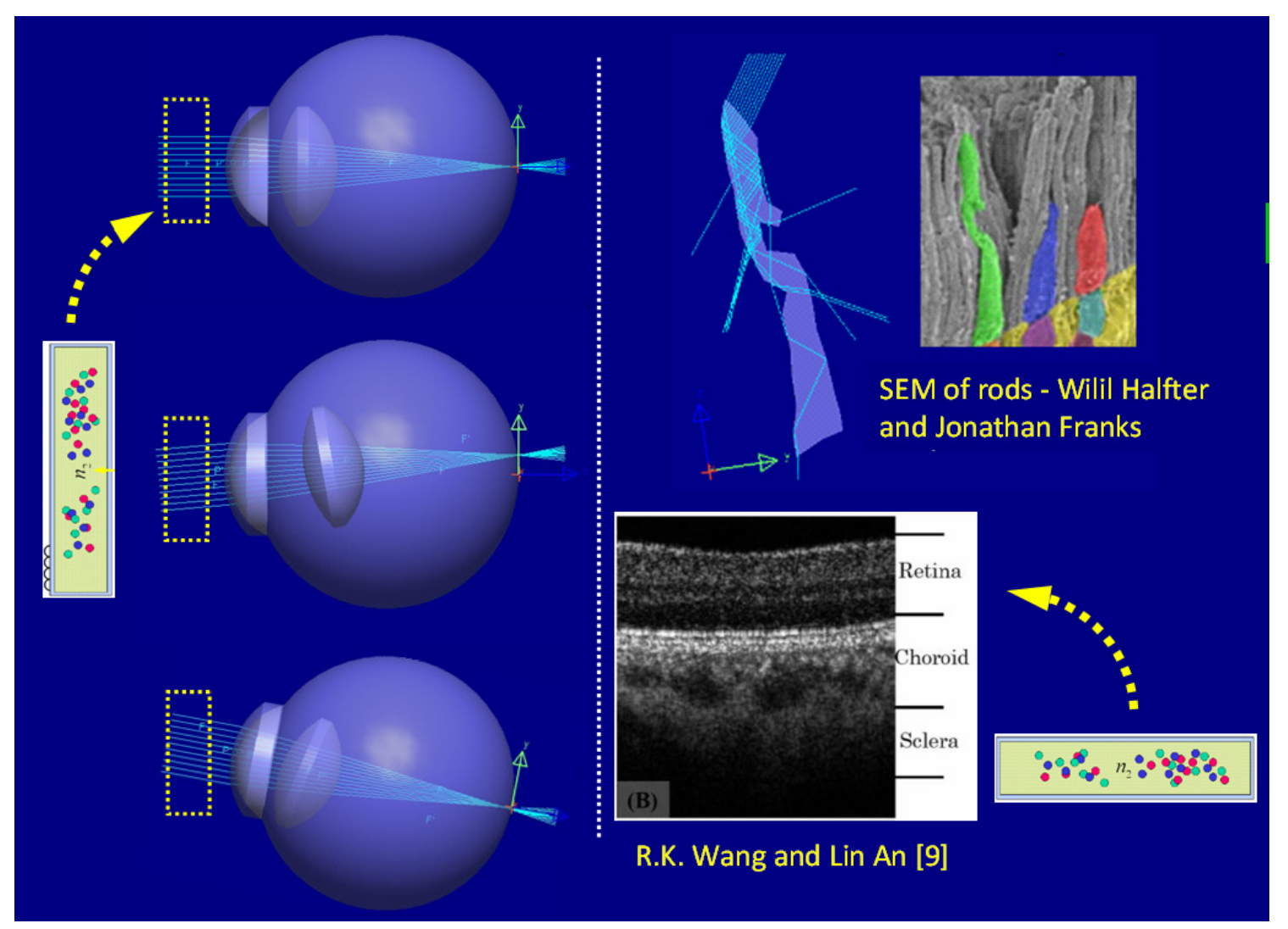

Figure 4. Modelling the Emitting radiant for visual science - Left: Wearable device outside the human eye; Right: Layer structures [9] in the retina part can be modelled to understand the photon intensity distribution within layers 


\section{Conclusions}

Simulation of the optical layer structure with microstructure on the emitting surface is critical for next generation Bio-photonics components. Through the present discourse, one can reason out that the combination of the textures, refractive layer; immersed and Boolean operations can represent several kinds of emitting patterns. The end of this report is to complete the integration between the present methods and the 3D printing for visual information and associated bio-compatible applications.

\section{Acknowledgements}

The authors would like to appreciate the financial support for this research from National Science Council and Minister of Science and Technology under grant no. NSC101-2221-E-164-014 and MOST 103-2221-E-164 -002. Mr. Pei-Yu Lin, Jun-Yu Chen and Yu-Kai Tsai are undergraduate students for the optical simulations are also appreciated.

\section{REFERENCES}

[1] S. Kataria, S. Natarajan, N. Agarwala, 2001, Optical tomographic evaluation of retinal nerve fiber layer in glaucomatous eyes, J. Bombay Ophth. Assoc. 11(3), 99-103.
[2] V. V. Tuchin , 2005, Optical clearing of tissues and blood using the immersion method, J. Phys. D: Appl. Phys. 38 2497.

[3] E. V. Koblova, A. N. Bashkatov, L. E. Dolotov, Y. P. Sinichkin, T. G. Kamenskikh, E. A. Genina and V. V. Tuchin, 2007, Monte Carlo modeling of eye iris color, Proc. of SPIE 6535, 653521-10

[4] H. Hickey, 2008, Contact lenses with circuits, lights a possible platform for superhuman vision, University of Washington archived article Jan. 17

[5] G. Sęk, P. Podemski, J. Andrzejewski, J. Misiewicz, S. Hein, S. Höfling and A. Forchel, 2009, Immersion layer in columnar quantum dash structure as a polarization insensitive light emitter at $1.55 \mu \mathrm{m}$, Appl. Phys. Express 2 061102 .

[6] J. Joab, 2010, Bionic Contact Lens May Create Tiny Personal Displays, National Geographic News, http://news.nationalgeographic.com/news/2008/01/080129-b ionic-eye.html

[7] P. A. d. Santos, 2013, Influence of light source and immersion media in surface roughness and hardness of a nano filled composite resin, Journal of Research in Dentistry $1(2), 166-174$.

[8] S. McGoldrick, 2013, Bionic Lens: A Look into the Future, Optical Prism, March Issue 30(8), 18-20.

[9] R. K. Wang and Lin An, 2011, Multifunctional imaging of human retina and choroids with $1050-\mathrm{nm}$ spectral domain optical coherence tomography at $92-\mathrm{kHz}$ line scan rate, $J$. Biomed. Opt. 16(5), 050503-1 3. 